\title{
Phylogenetic Analysis of Hemagglutinin Genes of H9N2 Avian Influenza Viruses Isolated from Chickens in Shandong, China, between 1998 and 2013
}

\author{
Yuxin Zhao, ${ }^{1}$ Song Li, ${ }^{2}$ Yufa Zhou, ${ }^{3,4}$ Wengang Song, ${ }^{2}$ Yujing Tang, \\ Quanhai Pang, ${ }^{3}$ and Zengmin Miao ${ }^{1}$ \\ ${ }^{1}$ College of Life Sciences, Taishan Medical University, Taian 271000, China \\ ${ }^{2}$ College of Basic Medicine, Taishan Medical University, Taian 271000, China \\ ${ }^{3}$ College of Animal Science and Technology, Shanxi Agricultural University, Taigu 030800, China \\ ${ }^{4}$ Animal Disease Control Center, Animal Husbandry Bureau of Daiyue, Taian 271000, China \\ Correspondence should be addressed to Zengmin Miao; zengminmiao@126.com
}

Received 22 June 2015; Accepted 31 August 2015

Academic Editor: Gregory Tannock

Copyright ( 2015 Yuxin Zhao et al. This is an open access article distributed under the Creative Commons Attribution License, which permits unrestricted use, distribution, and reproduction in any medium, provided the original work is properly cited.

\begin{abstract}
Since H9N2 avian influenza virus (AIV) was first isolated in Guangdong province of China, the virus has been circulating in chicken flocks in mainland China. However, a systematic phylogenetic analysis of H9N2 AIV from chickens in Shandong of China has not been conducted. Based on hemagglutinin (HA) gene sequences of H9N2 AIV s isolated from chickens in Shandong of China between 1998 and 2013, genetic evolution of $35 \mathrm{HA}$ gene sequences was systematically analyzed in this study. Our findings showed that the majority of H9N2 AIVs (21 out of 35) belonged to the lineage h9.4.2.5. Most of isolates (33 out of 35) had a PSRSSR $\downarrow$ GLF motif in HA cleavage site. Importantly, 29 out of these 35 isolates had an amino acid exchange (Q226L) in the receptor-binding site. The substitution showed that H9N2 AIVs had the potential affinity to bind to human-like receptor. The currently prevalent H9N2 AIVs in Shandong belonged to the lineage h9.4.2.5 which are different from the vaccine strain SS/94 clade h9.4.2.3. Therefore, the long-term surveillance of H9N2 AIVs is of significance to combat the possible H9N2 AIV outbreaks.
\end{abstract}

\section{Introduction}

Since H9N2 subtype avian influenza virus (AIV) was first isolated in Guangdong province of China in 1994, this subtype influenza virus has quickly spread to other areas of China and become prevalent in poultry [1-3]. H9N2 AIV infections in chicken flocks have led to massive economic losses due to egg production decline or high mortality related with coinfection with other pathogens [4-6].

Although there is no evidence for human-to-human transmission of H9N2 AIV, several human infections with H9N2 AIV have been reported [7-11]. It is noteworthy that the number of humans infected by H9N2 AIV in serological surveillance, especially poultry workers, was much higher than that of the confirmed cases $[12,13]$. H9N2 AIV is able to reassort with other subtypes of influenza virus, including H6N1, H6N2, H5N1, and H7N9. The increased risk of animalto-human spread with avian viruses that have the H9N2 internal genes has posed a greater pandemic threat [14-16].

Since the late 1990s, vaccination has been carried out in China to prevent and control H9N2 AIV infection in chicken flocks. Commercial vaccines did not provide complete protection for the endemic H9N2 AIVs in China. Up to the present time, H9N2 virus has regularly been found in vaccinated chickens in Shandong of China $[1,17,18]$. Therefore, this study was designed to perform a comprehensive phylogenetic analysis of H9N2 AIVs from chickens in Shandong of China to provide an important guidance for the effective control of H9N2 AIV infection. 
TABLE 1: HA genes of 35 H9N2 AIVs isolated from Shandong of China from 1998 to 2013.

\begin{tabular}{|c|c|c|c|}
\hline Strains & Abbreviation & Date of isolation & GenBank accession number \\
\hline A/chicken/Shandong/WF/1998(H9N2) & $\mathrm{Ck} / \mathrm{SD} / \mathrm{WF} / 98$ & 1998 & JF795136 \\
\hline A/chicken/Shandong/B1/1998(H9N2) & $\mathrm{Ck} / \mathrm{SD} / \mathrm{B} 1 / 98$ & 1998 & EU573939 \\
\hline A/chicken/Shandong/JN/1999(H9N2) & $\mathrm{Ck} / \mathrm{SD} / \mathrm{JN} / 99$ & 1999 & HM773437 \\
\hline A/chicken/Shandong/LW/1999(H9N2) & $\mathrm{Ck} / \mathrm{SD} / \mathrm{LW} / 99$ & 1999 & HM773438 \\
\hline A/chicken/Shandong/241/2001(H9N2) & $\mathrm{Ck} / \mathrm{SD} / 241 / 01$ & 2001 & KF746827 \\
\hline A/chicken/Shandong/3/2001(H9N2) & $\mathrm{Ck} / \mathrm{SD} / 3 / 01$ & 2001 & KF313566 \\
\hline A/chicken/Shandong/244/2002(H9N2) & $\mathrm{Ck} / \mathrm{SD} / 244 / 02$ & 2002 & KF746810 \\
\hline A/chicken/Shandong/BZ02/2002(H9N2) & $\mathrm{Ck} / \mathrm{SD} / \mathrm{BZ} 02 / 02$ & 2002 & JQ710462 \\
\hline A/chicken/Shandong/2/2003(H9N2) & $\mathrm{Ck} / \mathrm{SD} / 2 / 03$ & 2003 & FJ190117 \\
\hline A/chicken/Shandong/S2/2005(H9N2) & $\mathrm{Ck} / \mathrm{SD} / \mathrm{S} 2 / 05$ & 2005 & HM773440 \\
\hline A/chicken/Shandong/JN5/2005(H9N2) & $\mathrm{Ck} / \mathrm{SD} / \mathrm{JN} 5 / 05$ & 2005 & HM773436 \\
\hline A/chicken/Shandong/JN4/2005(H9N2) & $\mathrm{Ck} / \mathrm{SD} / \mathrm{JN} 4 / 05$ & 2005 & HM773435 \\
\hline A/chicken/Shandong/ZC820/2006(H9N2) & $\mathrm{Ck} / \mathrm{SD} / \mathrm{ZC} 820 / 06$ & 2006 & FJ190135 \\
\hline A/chicken/Shandong/YB06/2006(H9N2) & $\mathrm{Ck} / \mathrm{SD} / \mathrm{YB} 06 / 06$ & 2006 & JQ710463 \\
\hline A/chicken/Shandong/LX414/2007(H9N2) & $\mathrm{Ck} / \mathrm{SD} / \mathrm{LX} 414 / 07$ & 2007 & FJ190141 \\
\hline A/chicken/Shandong/LX331/2007(H9N2) & $\mathrm{Ck} / \mathrm{SD} / \mathrm{LX} 331 / 07$ & 2007 & FJ190140 \\
\hline A/chicken/Shandong/LX327/2007(H9N2) & $\mathrm{Ck} / \mathrm{SD} / \mathrm{LX} 327 / 07$ & 2007 & FJ190139 \\
\hline A/chicken/Shandong/SD0014/2008(H9N2) & $\mathrm{Ck} / \mathrm{SD} / \mathrm{SD} 0014 / 08$ & 2008 & HQ398361 \\
\hline A/chicken/Shandong/1/2008(H9N2) & $\mathrm{Ck} / \mathrm{SD} / 1 / 08$ & 2008 & HQ326722 \\
\hline A/chicken/Shandong/LX316/2008(H9N2) & $\mathrm{Ck} / \mathrm{SD} / \mathrm{LX} 316 / 08$ & 2008 & FJ190138 \\
\hline A/chicken/Shandong/BD/2009(H9N2) & $\mathrm{Ck} / \mathrm{SD} / \mathrm{BD} / 09$ & 2009 & HM773434 \\
\hline A/chicken/Shandong/SG2/2009(H9N2) & $\mathrm{Ck} / \mathrm{SD} / \mathrm{SG} 2 / 09$ & 2009 & HM751194 \\
\hline A/chicken/Shandong/SG1/2009(H9N2) & $\mathrm{Ck} / \mathrm{SD} / \mathrm{SG} 1 / 09$ & 2009 & HM751186 \\
\hline A/chicken/Shandong/YT1/2010(H9N2) & $\mathrm{Ck} / \mathrm{SD} / \mathrm{YT} 1 / 10$ & 2010 & KJ419948 \\
\hline A/chicken/Shandong/YT2/2010(H9N2) & $\mathrm{Ck} / \mathrm{SD} / \mathrm{YT} 2 / 10$ & 2010 & KJ419946 \\
\hline A/chicken/Shandong/YT4/2010(H9N2) & $\mathrm{Ck} / \mathrm{SD} / \mathrm{YT} 4 / 10$ & 2010 & KJ419943 \\
\hline A/chicken/Shandong/BD1/2011(H9N2) & $\mathrm{Ck} / \mathrm{SD} / \mathrm{BD} 1 / 11$ & 2011 & KJ419954 \\
\hline A/chicken/Shandong/YT15/2011(H9N2) & $\mathrm{Ck} / \mathrm{SD} / \mathrm{YT} 15 / 11$ & 2011 & KJ419953 \\
\hline A/chicken/Shandong/YT1/2011(H9N2) & $\mathrm{Ck} / \mathrm{SD} / \mathrm{YT} 1 / 11$ & 2011 & KJ419947 \\
\hline A/chicken/Shandong/YS/2012(H9N2) & $\mathrm{Ck} / \mathrm{SD} / \mathrm{YS} / 12$ & 2012 & KC879302 \\
\hline A/chicken/Shandong/WF4/2012(H9N2) & $\mathrm{Ck} / \mathrm{SD} / \mathrm{WF} 4 / 12$ & 2012 & JX448768 \\
\hline A/chicken/Shandong/P6/2012(H9N2) & $\mathrm{Ck} / \mathrm{SD} / \mathrm{P} 6 / 12$ & 2012 & JX448767 \\
\hline A/chicken/Shandong/HK1/2013(H9N2) & $\mathrm{Ck} / \mathrm{SD} / \mathrm{HK} 1 / 13$ & 2013 & KJ419952 \\
\hline A/chicken/Shandong/LC1/2013(H9N2) & $\mathrm{Ck} / \mathrm{SD} / \mathrm{LC} 1 / 13$ & 2013 & KJ419951 \\
\hline A/chicken/Shandong/LC2/2013(H9N2) & $\mathrm{Ck} / \mathrm{SD} / \mathrm{LC} 2 / 13$ & 2013 & KJ419950 \\
\hline
\end{tabular}

\section{Materials and Methods}

2.1. Viruses. A total of $35 \mathrm{HA}$ gene sequences of $\mathrm{H} 9 \mathrm{~N} 2$ AIVs isolated from chickens in Shandong of China between 1998 and 2013 were obtained from the National Center for Biotechnology Information (NCBI) Influenza Viruses Resource (http://www.ncbi.nlm.nih.gov/genomes/FLU/FLU .html) (Table 1).

2.2. Phylogenetic Analysis. Multiple sequence alignment was conducted using ClustalW [19]. The phylogenetic tree was constructed using the neighbor-joining method with 1000 bootstrap replicates using MEGA 5.05 [20].

2.3. Antigenic Analysis. Antigenic analysis of H9N2 AIV was conducted using hemagglutination inhibition (HI) to assess antigenic relationship between the emerging H9N2 AIVs and the vaccine strain SS/94 [21]. According to the previously published work [22], 6-week-old specific pathogen-free (SPF) chickens were used to produce polyclonal antibodies against 7 H9N2 AIVs, including 6 isolates between 2012 and 2013, and the vaccine strain SS/94.

\section{Results}

3.1. Homology Analysis. The coding sequences of HA gene of $35 \mathrm{H} 9 \mathrm{~N} 2 \mathrm{AIV}$ s in this study contained 1,683 nucleotides. HA gene nucleotide sequences and deduced amino acid (AA) sequence identities among the 35 tested strains ranged from 84.0 to $100.0 \%$ and 89.1 to $100.0 \%$, respectively. Compared with three vaccine strains, the homologies of HA nucleotide 
TABLE 2: Similarity analysis of HA nucleotide sequence and deduced amino acid sequence of H9N2 AIVs and vaccine strains.

\begin{tabular}{lccc}
\hline & Virus & Nucleotide sequence & Amino acid sequence \\
\hline & 35 H9N2 AIVs in this study & $84.0-100.0 \%$ & $89.1-100.0 \%$ \\
Vaccine strains & A/chicken/Guangdong/SS/1994 & $86.2-92.1 \%$ & $83.6-87.5 \%$ \\
& A/chicken/Shandong/6/1996 & $84.5-91.4 \%$ & $86.7-89.2 \%$ \\
A/chicken/Shanghai/F/1998 & $84.0-93.0 \%$ & $87.7-92.2 \%$ \\
\hline
\end{tabular}

sequences and AA sequence entities of the 35 H9N2 AIVs were $84.0-93.0 \%$ and $83.6-92.2 \%$, respectively (Table 2 ).

3.2. Phylogenetic Analysis. All of $35 \mathrm{H} 9 \mathrm{~N} 2$ AIVs belonged to the lineage h9.4.2 represented by Y280 or BJ194. The lineage h9.4.2 is divided into 6 tertiary levels: h9.4.2.1, h9.4.2.2, h9.4.2.3, h9.4.2.4, h9.4.2.5, and h9.4.2.6 [23]. The majority of H9N2 AIVs (21 out of 35) belonged to the lineage h9.4.2.5 represented by strains Ck/ZJ/HE6/09 and Ck/GX/55/05 [24]. All of 12 H9N2 AIVs isolated between 2010 and 2013 belonged to the lineage h9.4.2.5. Vaccine strains SS/94 and 6/96 belonged to the linage h9.4.2.3, and vaccine strain F/98 was clustered in the lineage h9.4.2.1 (Figure 1).

3.3. Important Site Analysis of Deduced AA Sequences. The cleavage sites of precursor HA protein of H9N2 AIVs showed two different motifs in this study. 33 H9N2 AIVs isolated between 1999 and 2013 had a PSRSSR $\downarrow$ GLF motif, which can meet the characteristic of low-pathogenic avian influenza virus (Table 3 ).

AA residues at receptor-binding site of $\mathrm{HA}$ protein were conserved among the 35 H9N2 AIVs. Only one substitution was found in the position $\mathrm{A} 190 \mathrm{~V}(\mathrm{~T})$ (H3 numbering is used throughout the paper) (Table 3 ). On the right edge of receptor-binding pocket, $\mathrm{AA}$ sequences were conserved and no substitution was found in this study. AA residues on the left edge of receptor-binding pocket had a substitution Q227L (M), and most isolates (4 out of 6) between 2012 and 2013 had $\mathrm{M}$ at position 227. 29 out of $35 \mathrm{H} 9 \mathrm{~N} 2$ AIVs had leucine (L) at position 226, including all of the isolates between 2005 and 2013 (Table 3).

3.4. Antigenic Analysis. HI test was used to evaluate protection efficacy of commercial vaccine strain SS/94 widely used in Shandong against 6 H9N2 AIVs isolated in Shandong between 2012 and 2013. The results showed that antisera against $6 \mathrm{H} 9 \mathrm{~N} 2 \mathrm{AIVs}$ (Ck/SD/YS/12, Ck/SD/WF4/12, Ck/SD/ $\mathrm{P} 6 / 12, \mathrm{Ck} / \mathrm{SD} / \mathrm{HK} 1 / 13, \mathrm{Ck} / \mathrm{SD} / \mathrm{LC} 1 / 13$, and $\mathrm{Ck} / \mathrm{SD} / \mathrm{LC} 2 / 13$ ) reacted well with the emerging $\mathrm{H} 9 \mathrm{~N} 2 \mathrm{AIVs}$ ( $\mathrm{HI}$ titer $\geq 1280$ ), but none of antisera against the emerging H9N2 AIVs reacted well with the vaccine strain SS/94 (HI titer $\leq 320)$ (Table 4).

\section{Discussion}

Vaccination is an effective way to prevent and control the spread of H9N2 AIVs, but the current vaccine used in Shandong is still prepared from the isolates in the early 1990s, and their protective efficacy has been decreasing $[3,18]$. HI test in this study showed that commercial vaccine strain SS/94 has not provided robust protection against the emerging H9N2 AIVs isolated in Shandong, China.

Previous investigation about phylogenetic analysis of HA genes of H9N2 AIVs isolated in China between 2008 and 2011 demonstrated that the lineage h9.4.2.5, as an emerging lineage, has become one of the dominant clades [22, 25]. Similarly, our findings showed that the lineage h9.4.2.5 was predominant between 2009 and 2013 in Shandong. The vaccine strain SS/94 belonged to the distinct lineage h9.4.2.3. Antigenic analysis also showed that the emerging viruses between 2012 and 2013 were antigenically different from the vaccine strain SS/94. Given the above results, it is of utmost importance to select novel candidate vaccine strains to combat the emerging H9N2 AIVs in Shandong of China.

On the left edge of receptor-binding pocket, most isolates (4 out of 6) between 2012 and 2013 had methionine (M) at position 227. But the vaccine strain SS/94 had glutamine (Q) at the same position. $33 \mathrm{H} 9 \mathrm{~N} 2 \mathrm{AIVs}$ isolated between 1999 and 2013 had a PSRSSR $\downarrow$ GLF motif in HA cleavage site; the vaccine strain SS/94 had a PAGSSR $\downarrow$ GLF motif. Additionally, 29 out of 35 H9N2 AIVs had leucine (L) at position 226, including all of the isolates between 2005 and 2013. The substitution indicated that the emerging H9N2 AIVs can preferentially bind to NeuAca2,6-Gal linkage and may have higher virulence $[3,26]$.

Our findings revealed that the currently prevalent H9N2 AIVs in Shandong belonged to the lineage h9.4.2.5 which are different from the vaccine strain SS/94 clade h9.4.2.3. Furthermore, $82.9 \%(29 / 35)$ of H9N2 AIVs contained L at position 226, indicating that these AIVs could cross species barrier to infect humans.

\section{Conclusion}

Taken together, genetic disparity between the emerging H9N2 AIVs and the current vaccine isolates should be taken into consideration when a control policy is established. In addition, the long-term surveillance of H9N2 AIVs is of significance to combat the possible H9N2 AIV outbreaks in chicken flocks.

\section{Conflict of Interests}

The authors declare that there is no conflict of interests regarding the publication of this paper. 


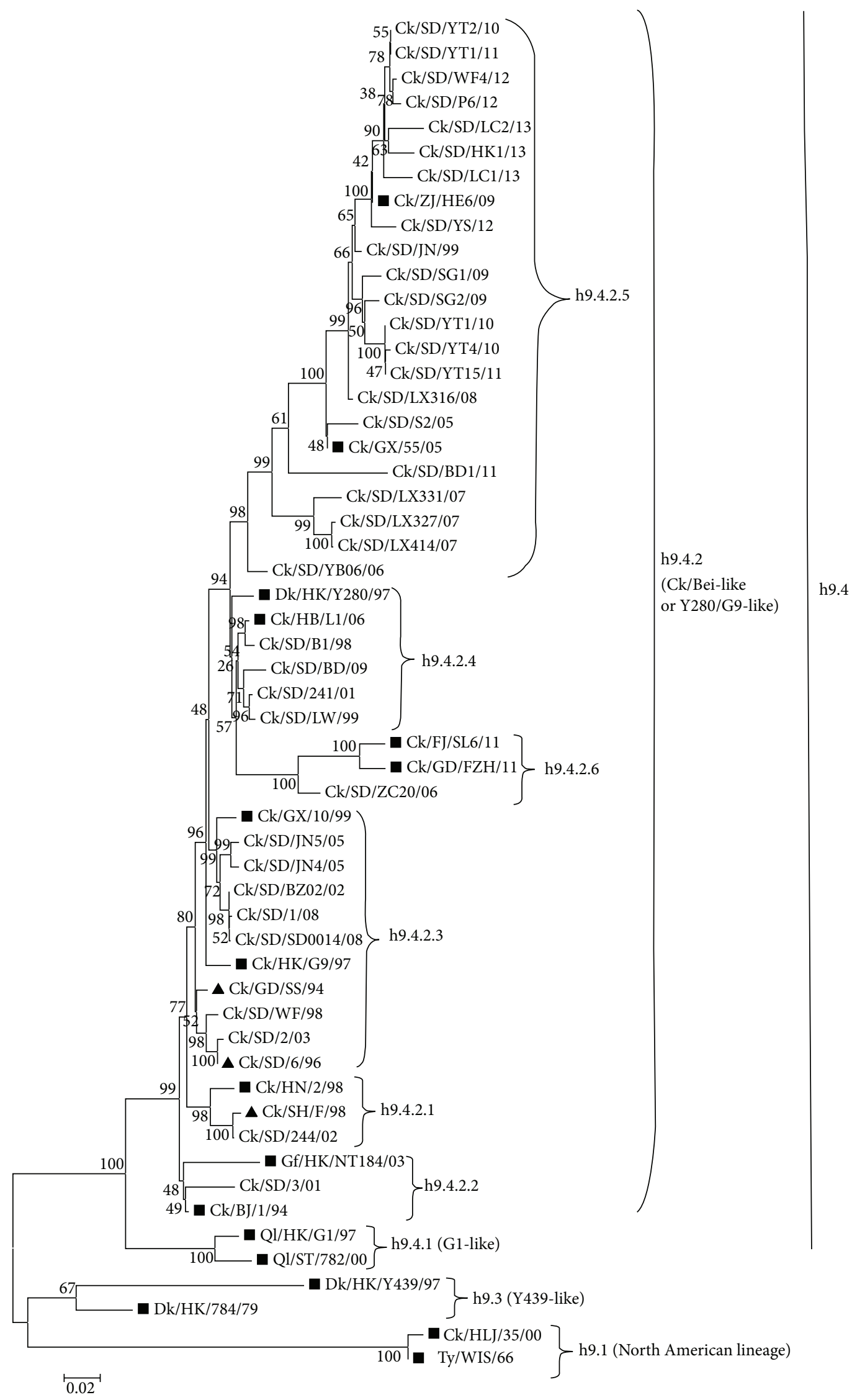

Figure 1: Phylogenetic tree of HA genes of H9N2 AIVs used in this study. The black squares indicate the representative strains of distinct H9 lineages. The black triangles indicate vaccine strains. 
TABLE 3: Amino acid sequence at the cleavage site and receptor-binding site of HA.

\begin{tabular}{|c|c|c|c|c|c|c|c|c|c|c|}
\hline \multirow{3}{*}{$\begin{array}{l}\text { Strain abbreviation } \\
\text { SD/6/96 }\end{array}$} & \multirow{2}{*}{$\begin{array}{l}\text { Left edge } \\
224-229\end{array}$} & \multicolumn{7}{|c|}{ Receptor-binding sites (RBS) } & \multirow{3}{*}{$\begin{array}{c}\text { Right edge } \\
\text { 134-138 } \\
\text { GTSKA } \\
\end{array}$} & \multirow{3}{*}{$\begin{array}{l}\text { Cleavage site } \\
\text { PARSSR } \downarrow \text { GLF }\end{array}$} \\
\hline & & 98 & 153 & 155 & 183 & 190 & 194 & 195 & & \\
\hline & NGQQGR & $\mathrm{P}$ & $\mathrm{W}$ & $\mathrm{T}$ & $\mathrm{N}$ & A & $\mathrm{L}$ & $\mathrm{Y}$ & & \\
\hline $\mathrm{SD} / \mathrm{WF} / 98$ & + & + & + & + & + & $\mathrm{T}$ & + & + & + & + \\
\hline $\mathrm{SD} / \mathrm{B} 1 / 98$ & + & + & + & + & + & $\mathrm{T}$ & + & + & + & + \\
\hline $\mathrm{SD} / \mathrm{JN} / 99$ & NGLQGR & + & + & + & + & + & + & + & + & PSRSSR $\downarrow$ GLF \\
\hline $\mathrm{SD} / \mathrm{LW} / 99$ & + & + & + & + & + & $\mathrm{V}$ & + & + & + & + \\
\hline $\mathrm{SD} / 241 / 01$ & + & + & + & + & + & $\mathrm{V}$ & + & + & + & + \\
\hline $\mathrm{SD} / 3 / 01$ & NGMQGR & + & + & + & + & $\mathrm{V}$ & + & + & + & + \\
\hline $\mathrm{SD} / 244 / 02$ & + & + & + & + & + & + & + & + & + & + \\
\hline $\mathrm{SD} / \mathrm{BZ} 02 / 02$ & + & + & + & + & + & $\mathrm{T}$ & + & + & + & + \\
\hline $\mathrm{SD} / 2 / 03$ & + & + & + & + & + & + & + & + & + & + \\
\hline $\mathrm{SD} / \mathrm{S} 2 / 05$ & NGLQGR & + & + & + & + & + & + & + & + & + \\
\hline $\mathrm{SD} / \mathrm{JN} 5 / 05$ & + & + & + & + & + & $\mathrm{V}$ & + & + & + & + \\
\hline $\mathrm{SD} / \mathrm{JN} 4 / 05$ & + & + & + & + & + & $\mathrm{V}$ & + & + & + & + \\
\hline SD/ZC820/06 & + & + & + & + & + & + & + & + & + & + \\
\hline SD/YB06/06 & + & + & + & + & + & $\mathrm{T}$ & + & + & + & + \\
\hline SD/LX414/07 & + & + & + & + & + & $\mathrm{V}$ & + & + & + & + \\
\hline SD/LX331/07 & + & + & + & + & + & + & + & + & + & + \\
\hline SD/LX327/07 & + & + & + & + & + & $\mathrm{T}$ & + & + & + & + \\
\hline $\mathrm{SD} / \mathrm{SD} 0014 / 08$ & + & + & + & + & + & $\mathrm{T}$ & + & + & + & + \\
\hline $\mathrm{SD} / 1 / 08$ & + & + & + & + & + & $\mathrm{V}$ & + & + & + & + \\
\hline SD/LX316/08 & + & + & + & + & + & $\mathrm{V}$ & + & + & + & + \\
\hline $\mathrm{SD} / \mathrm{BD} / 09$ & + & + & + & + & + & $\mathrm{V}$ & + & + & + & + \\
\hline $\mathrm{SD} / \mathrm{SG} 2 / 09$ & + & + & + & + & + & $\mathrm{T}$ & + & + & + & + \\
\hline $\mathrm{SD} / \mathrm{SG} 1 / 09$ & + & + & + & + & + & $\mathrm{T}$ & + & + & + & + \\
\hline $\mathrm{SD} / \mathrm{YT} 1 / 10$ & + & + & + & + & + & + & + & + & + & + \\
\hline $\mathrm{SD} / \mathrm{YT} 2 / 10$ & + & + & + & + & + & + & + & + & + & + \\
\hline SD/YT4/10 & + & + & + & + & + & + & + & + & + & + \\
\hline $\mathrm{SD} / \mathrm{BD} 1 / 11$ & + & + & + & + & + & $\mathrm{V}$ & + & + & + & + \\
\hline SD/YT15/11 & + & + & + & + & + & + & + & + & + & + \\
\hline $\mathrm{SD} / \mathrm{YT} 1 / 11$ & NGLMGR & + & + & + & + & $\mathrm{V}$ & + & + & + & + \\
\hline $\mathrm{SD} / \mathrm{YS} / 12$ & NGLQGR & + & + & + & + & $\mathrm{V}$ & + & + & + & + \\
\hline SD/WF4/12 & NGLMGR & + & + & + & + & $\mathrm{V}$ & + & + & + & + \\
\hline $\mathrm{SD} / \mathrm{P} 6 / 12$ & + & + & + & + & + & $\mathrm{V}$ & + & + & + & + \\
\hline $\mathrm{SD} / \mathrm{HK} 1 / 13$ & + & + & + & + & + & $\mathrm{V}$ & + & + & + & + \\
\hline $\mathrm{SD} / \mathrm{LC1} / 13$ & + & + & + & + & + & + & + & + & + & + \\
\hline $\mathrm{SD} / \mathrm{LC} 2 / 13$ & + & + & + & + & + & $\mathrm{T}$ & + & + & + & + \\
\hline
\end{tabular}

Note. Plus signs (+) indicate identical site.

TABLE 4: HI titers of the emerging H9N2 AIVs isolated in 2012 and 2013.

\begin{tabular}{|c|c|c|c|c|c|c|c|}
\hline \multirow{2}{*}{ Antigen } & \multicolumn{7}{|c|}{ Antisera $^{\mathrm{A}}$} \\
\hline & $\mathrm{SD} / \mathrm{YS} / 12$ & $\mathrm{SD} / \mathrm{WF} 4 / 12$ & $\mathrm{SD} / \mathrm{P} 6 / 12$ & $\mathrm{SD} / \mathrm{HK} 1 / 13$ & $\mathrm{SD} / \mathrm{LC1} / 13$ & $\mathrm{SD} / \mathrm{LC} 2 / 13$ & SS/94 \\
\hline SD/YS/12 & $10240^{B}$ & 2560 & 1280 & 1280 & 1280 & 1280 & 320 \\
\hline $\mathrm{SD} / \mathrm{WF} 4 / 12$ & 2560 & 10240 & 1280 & 2560 & 1280 & 2560 & 160 \\
\hline $\mathrm{SD} / \mathrm{P} 6 / 12$ & 2560 & 5120 & 2560 & 1280 & 5120 & 2560 & 320 \\
\hline SD/HK1/13 & 5120 & 10240 & 1280 & 10240 & 1280 & 2560 & 320 \\
\hline SD/LC1/13 & 2560 & 5120 & 5120 & 1280 & 5120 & 2560 & 160 \\
\hline SD/LC2/13 & 1280 & 2560 & 1280 & 2560 & 1280 & 10240 & 160 \\
\hline SS/94 & 320 & 320 & 40 & 160 & 320 & 40 & 1280 \\
\hline
\end{tabular}

Notes. ${ }^{\mathrm{A}}$ Antisera were tenfold diluted; ${ }^{\mathrm{B}}$ homologous tiers were marked in bold. 


\section{Authors' Contribution}

Yuxin Zhao, Song Li, and Yufa Zhou contributed equally to this work.

\section{Acknowledgments}

This study was supported by the National Natural Science Foundations of China (81501357, 81172791, and 81273212) and the Natural Science Foundation of Shandong (ZR2013CM030).

\section{References}

[1] C. Li, K. Yu, G. Tian et al., "Evolution of H9N2 influenza viruses from domestic poultry in Mainland China," Virology, vol. 340, no. 1, pp. 70-83, 2005.

[2] K. S. Li, K. M. Xu, J. S. M. Peiris et al., "Characterization of H9 subtype influenza viruses from the ducks of Southern China: a candidate for the next influenza pandemic in humans?" Journal of Virology, vol. 77, no. 12, pp. 6988-6994, 2003.

[3] Y. Sun and J. Liu, "H9N2 influenza virus in China: a cause of concern," Protein and Cell, vol. 6, no. 1, pp. 18-25, 2014.

[4] N. Kishida, Y. Sakoda, M. Eto, Y. Sunaga, and H. Kida, "Coinfection of Staphylococcus aureus or Haemophilus paragallinarum exacerbates H9N2 influenza A virus infection in chickens," Archives of Virology, vol. 149, no. 11, pp. 2095-2104, 2004.

[5] Q. Pan, A. Liu, F. Zhang et al., "Co-infection of broilers with Ornithobacterium rhinotracheale and H9N2 avian influenza virus," BMC Veterinary Research, vol. 8, article 104, 2012.

[6] K. M. Xu, G. J. D. Smith, J. Bahl et al., "The genesis and evolution of H9N2 influenza viruses in poultry from southern China, 2000 to 2005," Journal of Virology, vol. 81, no. 19, pp. 1038910401, 2007.

[7] K. M. Butt, G. J. D. Smith, H. Chen et al., "Human infection with an avian H9N2 influenza A virus in Hong Kong in 2003," Journal of Clinical Microbiology, vol. 43, no. 11, pp. 5760-5767, 2005.

[8] Y. Gou, J. Xie, and M. Wang, "A strain of influenza A H9N2 virus repeatedly isolated from human population in China," Chinese Journal of Experimental and Clinical Virology, vol. 14, no. 3, pp. 209-212, 2000.

[9] Y. Guo, J. Xie, K. Wu et al., "Characterization of genome of A/Guangzhou/333/99(H9N2) virus," Chinese Journal of Experimental and Clinical Virology, vol. 16, no. 2, pp. 142-145, 2002 (Chinese).

[10] T. Saito, W. Lim, T. Suzuki et al., "Characterization of a human H9N2 influenza virus isolated in Hong Kong," Vaccine, vol. 20, no. 1-2, pp. 125-133, 2001.

[11] T. M. Uyeki, Y.-H. Chong, J. M. Katz et al., "Lack of evidence for human-to-human transmission of avian influenza A (H9N2) viruses in Hong Kong, China, 1999," Emerging Infectious Diseases, vol. 8, no. 2, pp. 154-159, 2002.

[12] S. Liu, K. Ji, J. M. Chen et al., "Panorama phylogenetic diversity and distribution of type A influenza virus," PLoS ONE, vol. 4, no. 3, Article ID e5022, 2009.

[13] Q. Yu, L. Liu, J. Pu et al., "Risk perceptions for avian influenza virus infection among poultry workers, China," Emerging Infectious Diseases, vol. 19, no. 2, pp. 313-316, 2013.

[14] Y. Guan, K. F. Shortridge, S. Krauss, and R. G. Webster, "Molecular characterization of H9N2 influenza viruses: Were they the donors of the 'internal' genes of H5N1 viruses in Hong Kong?" Proceedings of the National Academy of Sciences of the United States of America, vol. 96, no. 16, pp. 9363-9367, 1999.

[15] D. Liu, W. Shi, and G. F. Gao, "Poultry carrying H9N2 act as incubators for novel human avian influenza viruses," The Lancet, vol. 383, no. 9920, p. 869, 2014.

[16] A. Wu, C. Su, D. Wang et al., "Sequential reassortments underlie diverse influenza H7N9 genotypes in China," Cell Host and Microbe, vol. 14, no. 4, pp. 446-452, 2013.

[17] Y. Sun, J. Pu, L. Fan et al., "Evaluation of the protective efficacy of a commercial vaccine against different antigenic groups of H9N2 influenza viruses in chickens," Veterinary Microbiology, vol. 156, no. 1-2, pp. 193-199, 2012.

[18] P. Zhang, Y. Tang, X. Liu et al., "Characterization of H9N2 influenza viruses isolated from vaccinated flocks in an integrated broiler chicken operation in eastern China during a 5 year period (1998-2002)," Journal of General Virology, vol. 89, no. 12, pp. 3102-3112, 2008.

[19] H. Yu, R.-H. Hua, Q. Zhang et al., "Genetic evolution of swine influenza A (H3N2) viruses in China from 1970 to 2006," Journal of Clinical Microbiology, vol. 46, no. 3, pp. 1067-1075, 2008.

[20] P. Zhang, Y. Tang, X. Liu et al., "A novel genotype H9N2 influenza virus possessing human $\mathrm{H} 5 \mathrm{~N} 1$ internal genomes has been circulating in poultry in eastern China since 1998," Journal of Virology, vol. 83, no. 17, pp. 8428-8438, 2009.

[21] S. Edwards, "OIE laboratory standards for avian influenza," Developments in Biologicals, vol. 124, pp. 159-162, 2006.

[22] H.-Q. Shen, Z.-Q. Yan, F.-G. Zeng et al., "Isolation and phylogenetic analysis of hemagglutinin gene of H9N2 influenza viruses from chickens in South China from 2012 to 2013," Journal of Veterinary Science, vol. 16, no. 3, pp. 317-324, 2015.

[23] J.-M. Chen, Y.-X. Sun, J.-W. Chen et al., "Panorama phylogenetic diversity and distribution of type A influenza viruses based on their six internal gene sequences," Virology Journal, vol. 6, article 137, 2009.

[24] R. Huang, A.-R. Wang, Z.-H. Liu et al., "Seroprevalence of avian influenza H9N2 among poultry workers in Shandong Province, China," European Journal of Clinical Microbiology and Infectious Diseases, vol. 32, no. 10, pp. 1347-1351, 2013.

[25] W. Jiang, S. Liu, G. Hou et al., "Chinese and global distribution of $\mathrm{H} 9$ subtype avian influenza viruses," PLoS ONE, vol. 7, no. 12, Article ID e52671, 2012.

[26] H. Wan and D. R. Perez, "Amino acid 226 in the hemagglutinin of H9N2 influenza viruses determines cell tropism and replication in human airway epithelial cells," Journal of Virology, vol. 81, no. 10, pp. 5181-5191, 2007. 

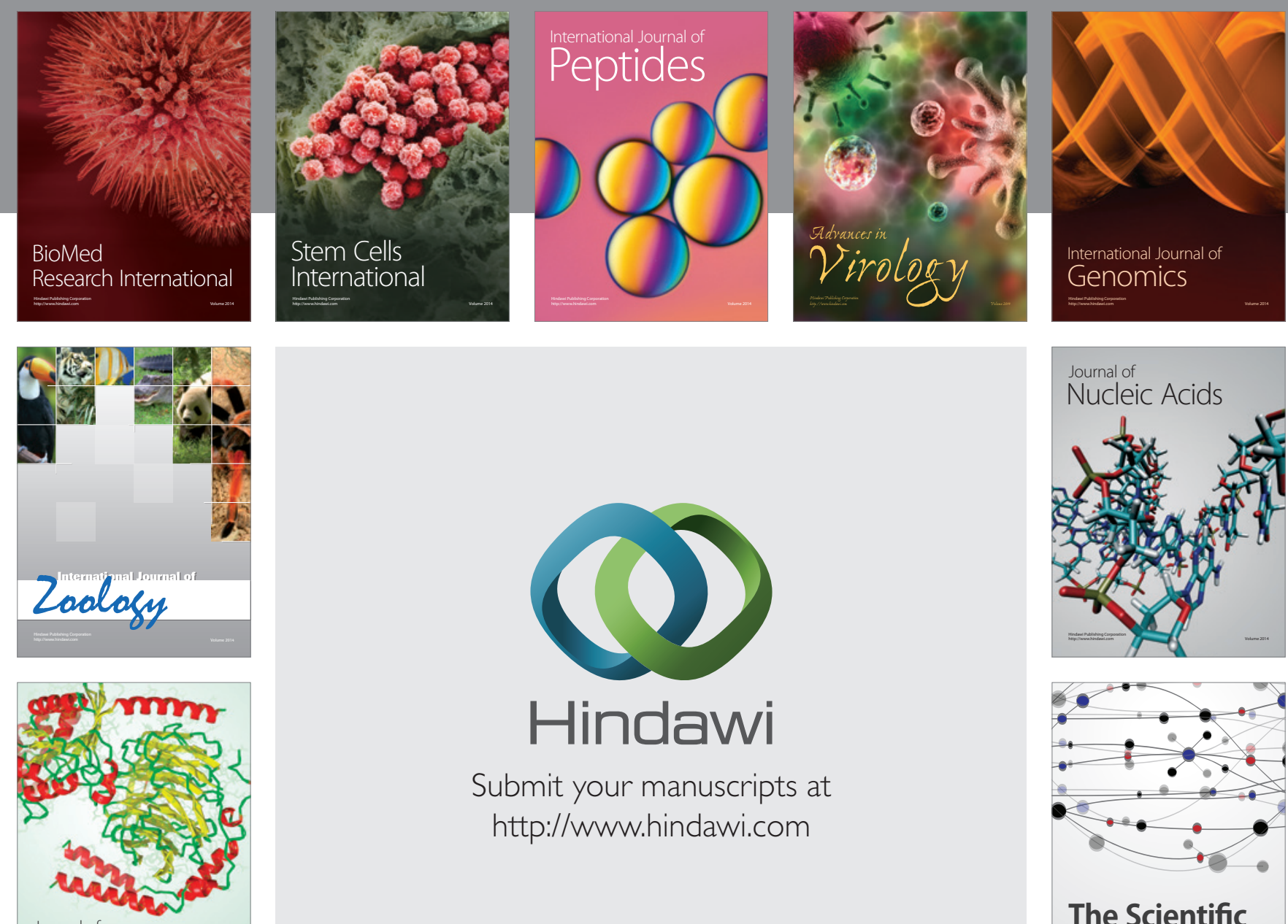

Submit your manuscripts at

http://www.hindawi.com

Journal of
Signal Transduction
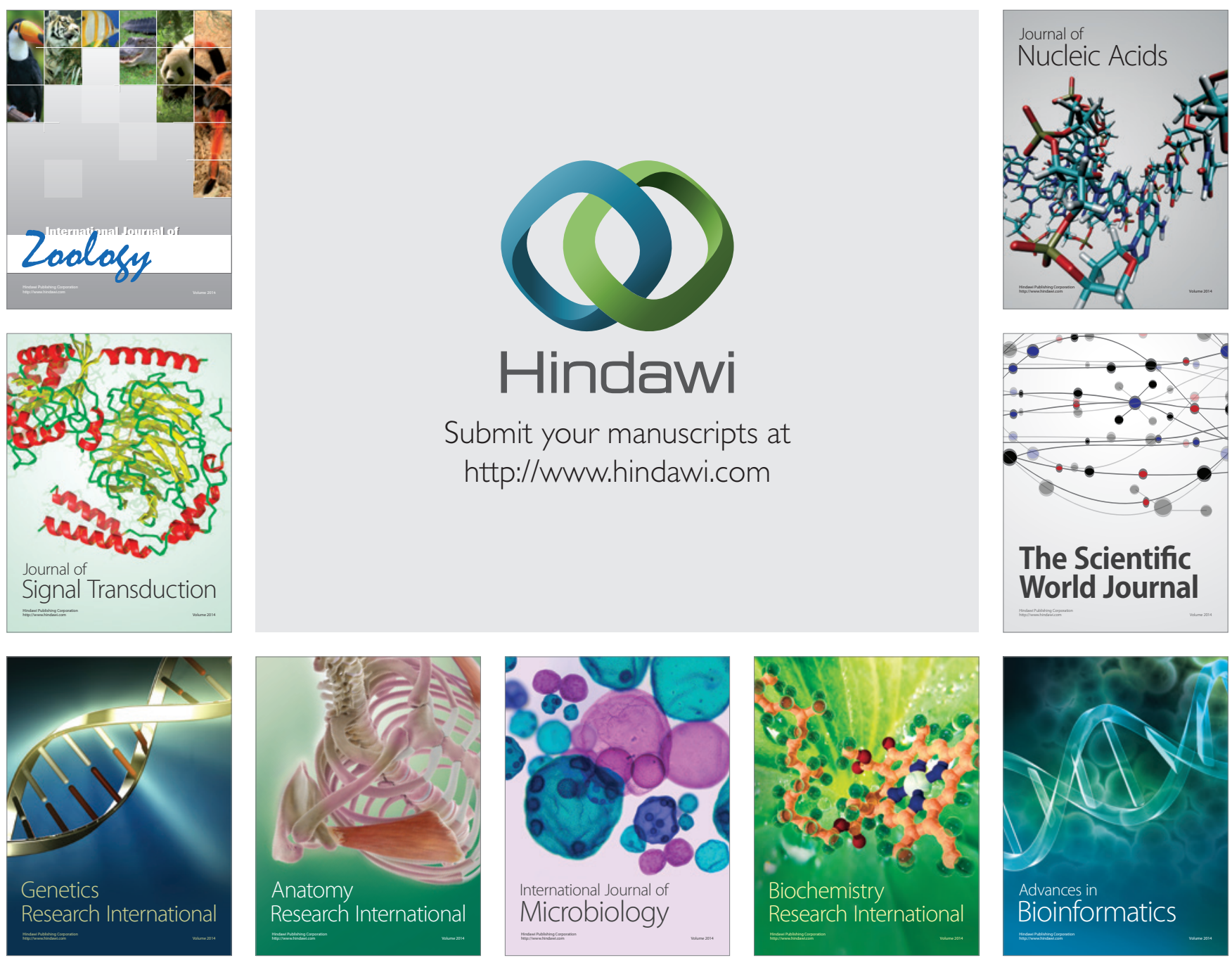

The Scientific World Journal
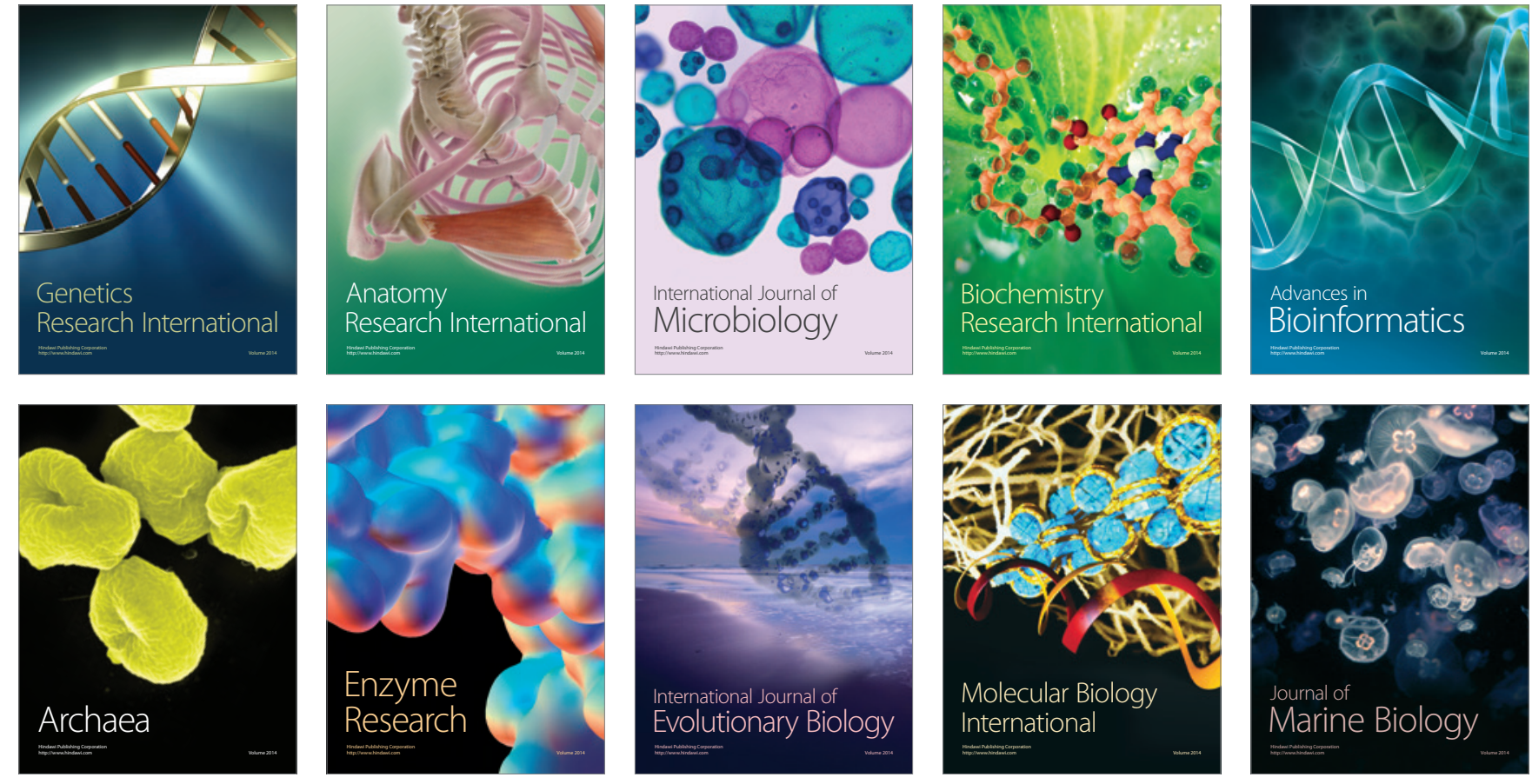